\title{
A Route Map for Wireless Sensor Networks over Large Coverage Area
}

\author{
Rishabh Jain, Partha Saha Adhikary, Guillermo Ramirez-Prado, Bashar Barmada, Iman Ardekani \\ Department of Computer Science \\ Unitec Institute of Technology \\ Auckland, New Zealand \\ \{gprado, bbarmada, iardekani\}@unitec.ac.nz
}

\begin{abstract}
This paper presents a mechanism to increase the coverage area of Wireless Sensor Networks (WSN) and increase the availability of wireless nodes. The proposed solution relies purely on open standards that implement 6LoWPAN and IPv6 to create a mesh network of wireless sensor nodes. Nodes in the mesh network build a chain connection from them to the border router to improve the coverage over a much wider area. In this proposed network, a Raspberry Pi, which hosts CETIC 6LBR, acts as a 6LoWPAN/RPL border router, and the mesh wireless sensor network is made out of several TI Tags using 6LoWPAN.
\end{abstract}

Keywords: Wireless Sensor Network, WSN coverage, multihop routing, 6LoWPAN.

\section{INTRODUCTION}

Several intelligent multi-sensor systems have emerged where continuous data collection is needed. Sensor nodes need to find the best path to connect to a sink, remain connected and publish signals continuously. Topology and routing is an open problem of Wireless Sensor Networks (WSN) [1], where smart sensor nodes may act as signal sources and routers. These configurable sensor nodes are deployed in quantity to sense the physical world surrounding them and to communicate among each other. The wireless channel imposes one of the biggest challenges that affects the efficiency of such smart system, as wireless channel is considered unpredictable and error prone, especially when the smart sensors are on move and the chance to lose their connection to the main network is high. A mechanism is needed to improve the coverage of WSN and extend the availability of the sensor nodes when it is possible.

The decision on how to define the topology of the wireless sensors relates to several factors including: How far nodes are from each other, the environment where they are placed, the energy consumption, the protocol used and even if the sensors are being added to the network one by one or all together when the WSN is setup. Several comparisons have been made among different setups in [2] [3] [4].

In this research we use Low-power Wireless Personal Area Networks (6LoWPAN) protocol, as it allows the network to have Internet identification for the nodes and Internet addresses. The evolution of 6LoWPAN and IPv6 has made it possible to access end devices such as wireless sensor nodes through a wide area [5]. This mesh network uses TI SensorTags, which can be configured to use 6LoWPAN, as wireless sensor nodes, and a Raspberry $\mathrm{Pi}$ as a network gateway to connect to the public network. The aim of proposed architecture is to allow a node that is outside the coverage area of the boarder router to continue its availability in the network and transmit its data to the border router through the nearest node available in the best possible way. In typical scenario, a wireless sensor node transmits signals out of the mesh through a direct connection to the border router, or though pre-defined path to a router node, which then routes

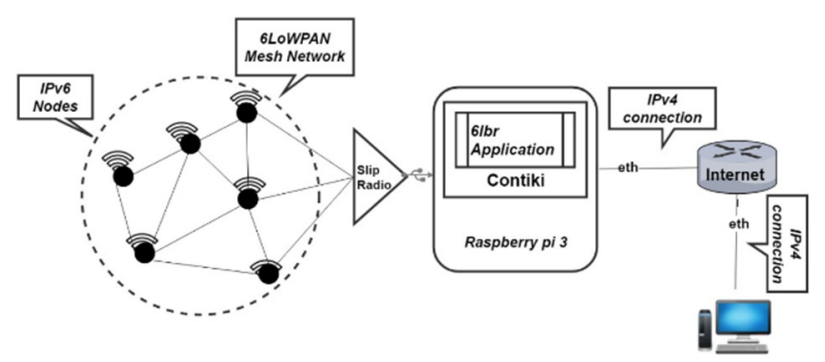

Fig. 1. The mesh of LoWPAN nodes is connected to the gateway through a slip radio node. The gateway supports 6LBR to map between the 6LoWPAN network and the Internet.

the data to the gateway. If a node is moved away and loses its connection to the border router or router node, it will be out of coverage and its data will not be collected. Data published while the node is out of coverage will be lost.

The proposed solution offers a flexible and adaptive mechanism to dynamically increase the coverage area of the network when nodes are moved. When a node loses connection to the border router, it searches for neighbor nodes in its local coverage area and determines the shortest path to reach the border router and chains itself to that neighbor. Every tag node has its own unique IPv6 address, and the communication between the nodes in the mesh network relies on Routing Protocol for Low power and Lossy Network (RPL) and Neighbor Discovery Protocol (NDP- internet protocol used with IPv6) [6]. One of the nodes acts as a slip radio to connect the rest of the mesh network to the gateway router. Every sensor node acts as a slave and a master to support uplink and downlink communications. A Raspberry Pi equipped with 6LoWPAN Border Router (6LBR) acts as an edge router to transmit data to cloud/server. It uses NAT64 for address translation between IPv6 and IPv4 addresses [7].

\section{THE PROPOSED SYSTEM}

Fig. 1 illustrates the proposed system design required for a wireless sensor network to route on a extended coverage area, which is in turn connected to an IPv4 Network.

The TI SensorTag nodes are configured to support IPv6 and together they form a 6LoWPAN mesh network. The default coverage area of a TI SensorTag node is 20 meters in diameter. In order to increase the coverage area of the TI SensorTag nodes each node acts as a 6LBR client and a CoAP server (CoAP is a specialized application layer protocol for constrained networks in Internet of Things) to communicate with other nodes in a client / server architecture. Each node may act as an MQTT client to be able to communicate with a broker (MQTT is a cloud protocol that sits over TCP protocol to send publication and subscription request to a cloud server). For the physical connection, each node uses universal asynchronous receiver-transmitter (UART) protocol to be 
Table 1. 6LoWPAN Border Router configuration to enable NAT64 and connect the mesh network with public network

\begin{tabular}{|l|l|}
\hline \multicolumn{1}{|c|}{ Parameter } & \multicolumn{1}{c|}{ Value } \\
\hline Internet protocol & IPv4, IPv6 \\
\hline IPv4 address & $192.168 .183 .170 / 24$ \\
\hline Public Gateway & 192.168 .183 .87 \\
\hline CoAP server port & 20000 \\
\hline HTTP server port & 25000 \\
\hline NAT64 & Enabled \\
\hline
\end{tabular}

\begin{tabular}{l|l|l|l|l|l|l|l|l} 
Node & Type & Web & Coap & Parent & $\begin{array}{l}\text { Up } \\
\text { PRR }\end{array}$ & $\begin{array}{l}\text { Down } \\
\text { PRR }\end{array}$ & $\begin{array}{l}\text { Last } \\
\text { seen }\end{array}$ & Status \\
\hline fdo0::212:4b00:1205:b80 & TI & web & coap & fe80::212:4b00:12b9:7505 & $100.0 \%$ & & 37 & OK \\
\hline fd00::212:4b00:12b9:7505 & TI & web & coap & fe80::212:4b00:1204:ff9a & $100.0 \%$ & & 46 & OK \\
\hline fd00::212:4b00:12b9:8203 & TI & web & coap & fe80::212:4b00:1204:ff9a & $100.0 \%$ & & 0 & OK \\
\hline
\end{tabular}

Fig. 2. Hierarchical links for the wireless sensor nodes.

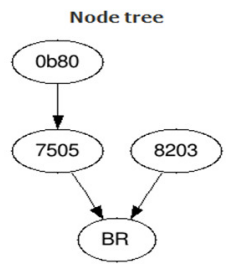

Fig. 3. Hierarchical links between nodes in the proposed system.

able to communicate with other nodes and routers in the network in a serial way.

Each node utilizes RPL (routing protocol for low-power and lossy network) [8] to create a routing table in each node and communicate between nodes. Technically, all these configurations are created on a separate file using Contiki OS and then flashed to the memory of these nodes.

The Slip Radio node in Fig. 1, is an intermediate point between the mesh network and border router. It helps the nodes to communicate with 6LBR and publish their data. In this network, one of the TI SensorTag node has been modified to work as a slip radio, and it has a permanent physical connection to the boarder router. The 6LoWPAN border router is Raspberry Pi 3 which is cloned with Contiki OS to support 6LBR application to receive communication from the 6LoWPAN mesh network, and NAT64 to communicate the public network and reach the cloud. Table 1 shows the main parameters used with the 6LoWPAN boarder router.

The Fig. 2. gives an example of the expected results after implementing the proposed system. The node column is the unique IPv6 address given to each node. The parent column identifies the parent for each node. Packet Reception Rate (PRR) evaluates the UDP channel. It checks the reliability of the connection. Therefore, UP and DOWN PRR indicates upstream and downstream PRR for each node. In Fig. 2. fd00: $: 212: 4 b 00: 1204: f f 9 a$ is the slip radio (BR) that acts as a parent for the other two nodes [7505 \& 8203], which are 20 meters away from the slip radio.

Fig. 3. Shows the hierarchical links between the nodes. Since $0 b 80$ node is outside the 20 meters range of the slip radio, and the mesh network, node 7505 , which is in range of node $0 b 80$, acts as its parent and allows it to be part of the mesh network. Consequently, increases the coverage area of the network beyond the default range of 20 meters.

In the extreme case, for $N$ nodes in the network, $(N-1)$ levels of hierarchical can be created in a chain format. Assuming that each node offers a coverage area of 20 meters, the overall coverage area of the network can reach up to $N \times 20$ meters.

\section{CONCLUSION}

The paper has presented a fixable 6LoWPAN mesh wireless sensor network that can extend the coverage area of the network in a scalable way. A boarder router, which supports $6 \mathrm{LBR}$, acts as a gateway between the mesh network and the public network, where data is routed though it to reach a cloud server. Each TI SensorTag node in the mesh network acts as a 6LBR Client, an MQTT Client, a CoAP server, and communicates with other nodes using Network-UART.

A slip radio node interfaces the mesh network with the border gateway. If a node moves away from the coverage area of the slip radio, it will search for the nearest neighbor node that is still part of the network and connect itself to that node. This chaining connection is extended over several levels between nodes, offering a flexibility in changing the topology of the network and preserve the availability of the nodes.

The system proposed in this paper can have a wide range of applications in real life such as, HealthCare, Greenhouse environment and smart houses. WSN have been proven effective for several applications, like agriculture and food industry [9] among others. Further security measures can be applied in the future to prevent malicious nodes from joining the mesh network and protect of the transmitted data.

\section{REFERENCES}

[1] K. M. D. Z. T. Sohraby, Wireless sensor networks: technology, protocols, and applications., John Wiley and Sons, 2007.

[2] I. Akyildiz, W. Su, Y. Sankarasubramaniam and E. Cayirci, "A survey on sensor networks," IEEE Communications Magazine, vol. 40, no. 8, pp. 102 - 114, 2002.

[3] J. Al-Karaki and A. Kamal, "Routing techniques in wireless sensor networks: a survey," IEEE Wireless Communications, vol. 11, no. 6, pp. $6-28,2004$

[4] M. S. Al-Fares, Z. Sun and H. Cruickshank, "A Hierarchical Routing Protocol for," Proceedings of the International MultiConference of Engineers and Computer Scientists, vol. 1, 2009.

[5] G. Mulligan, "The 6LoWPAN architecture," in EmNets '07 Proceedings of the 4th workshop on Embedded networked sensors, Cork, Ireland, 2007.

[6] Y. X. S. W. T. L. Ming Liu, "Design and Optimization of Hierarchical Routing Protocol for 6LoWPAN," International Journal of Distributed Sensor Networks, vol. 11, no. 5, 2015.

[7] M. Bagnulo, P. Mathews and I. v. Beijnum, "Stateful NAT64 Network Address and Protocol Translation from IPv6 Clients to IPv4 Servers," Internet Engineering Task Force (IETF), p. 50, April, 2011.

[8] E. P. Thubert, A. Brandt, J. Hui, R. Kelsey, P. Levis, K. Pister, R. Struik, J. Vasseur and R. Alexander, " RPL: IPv6 Routing Protocol for Low-Power and Lossy Networks," Internet Engineering Task Force (IETF) , p. 157, March 2012.

[9] N. Z. M. W. Ning Wang, "Wireless sensors in agriculture and food industry-Recent development and future perspective," Computers and Electronics in Agriculture, vol. 50, no. 1, pp. 1-14, 2006. 